\title{
Acute severe asthma treated by mechanical ventilation: 10 years' experience from a district general hospital
}

\author{
AR LUKSZA, P SMITH, J COAKLEY, IJ GORDAN, ST ATHERTON \\ From the Intensive Care Unit and Clinical Research Laboratory, Whiston Hospital, Prescot, Merseyside
}

ABSTRACT A retrospective study was made of patients requiring mechanical ventilation (intermittent positive pressure ventilation-IPPV) for acute severe asthma in a district general hospital during 1974-83. Thirty two patients required IPPV on 34 occasions. Complications included pneumothorax in six $(18 \%)$ patients, chest infection in $12(35 \%)$ patients, pulmonary collapse in three $(9 \%)$ patients, hypotension at induction of IPPV in $12(35 \%)$, and gastrointestinal bleeding in three $(9 \%)$. Three $(9 \%)$ patients died. Therapeutic bronchial lavage was performed in 19 patients. The procedure produced a rise in effective static compliance from a mean of 17 (SD 6) $\mathrm{ml} / \mathrm{cm}_{2} \mathrm{O}$ to $24(9) \mathrm{ml} / \mathrm{cm} \mathrm{H}_{2} \mathrm{O}$ at four hours. Bronchial lavage was associated with a significant excess of respiratory infections. A feature common to most patients was undertreatment before admission to hospital.

\begin{abstract}
Although the hospital mortality from acute severe asthma is less than $1 \%^{1}$ mechanical ventilation (intermittent positive pressure ventilation: IPPV) is life saving in a few patients. We have reviewed the efficacy and safety of IPPV and therapeutic bronchial lavage in the management of acute severe asthma in a district general hospital. Furthermore, we have analysed the pattern of asthma before IPPV in an attempt to identify preventable factors.
\end{abstract}

\section{Methods}

A retrospective study of the hospital case notes and the intensive care unit records was undertaken in respect of all patients admitted to the intensive care unit at Whiston Hospital because of acute severe asthma in the period 1974-83. The indications for admitting patients to the intensive care unit were based on the Jones index and have remained unchanged since $1972 .^{2}$ Thus patients with grades 3 and 4 asthma or grade $2 b$ of longer than eight hours' duration were treated in the intensive care unit. Grade $2 \mathrm{~b}$ refers to patients who are largely confined to a chair or bed and can get up only with great difficulty. Grades 3 and 4 refer to patients immobilised by their disease, the distinguishing feature being exhaustion in

Address for reprint requests: Dr AR Luksza, Furness General Hospital, Barrow-in-Furness, Cumbria LA14 4LF.

Accepted 8 October 1985 the latter group. In all grades sleep is regularly disturbed and bronchodilator treatment by inhaler only partly effective.

Initial treatment comprised treatment with humidified oxygen, rehydration, nebulised salbutamol $5 \mathrm{mg}$ every four hours administered by intermittent positive pressure breathing with a Bird ventilator, and an intravenous infusion of aminophylline at a rate of $0.9 \mathrm{mg} / \mathrm{kg}$ per hour. Corticosteroid treatment was used in conventional doses (hydrocortisone 100-200 mg two hourly) until 1978, when a trial of its efficacy was undertaken. ${ }^{3}$ Antibiotics were not given routinely, but careful bacteriological monitoring, including daily sputum culture, was carried out. The indications for starting IPPV were either immediate or delayed. Immediate indications comprised impaired consciousness, hypoxic confusion, cardiorespiratory arrest, or exhaustion, with or without hypercapnia (grade 4 asthma). Where IPPV was delayed, the indication was a worsening clinical trend despite conventional treatment, as judged by increasing heart rate, decreasing peak expiratory flow (PEF), worsening gas exchange, and exhaustion.

The treatment was explained to the patient and informed consent obtained where possible. A nasogastric tube was passed and the stomach contents were aspirated. Anaesthesia was induced with thiopentone or diazepam together with suxamethonium. A cuffed oral endotracheal tube was inserted. Sub- 
sequently phenoperidine was used for sedation and pancuronium as a muscle relaxant. A chest radiograph was taken immediately to check the position of the endotracheal tube and to exclude pneumothorax. Ten millilitres of aluminium hydroxide were instilled into the nasogastric tube every two hours in an attempt to reduce the risks of stress ulceration. Initial ventilator settings included a slow respiratory rate (10-12/min), a preset tidal volume (VT) of $400-500$ $\mathrm{ml}$, and high inspired oxygen concentration. Hypotension at the time of induction was common and was corrected by rapid intravenous infusion of colloid solutions. Once the blood pressure had been corrected, VT was increased to achieve normocapnic ventilation. In the more severe cases, peak ventilator pressures in excess of $50 \mathrm{~cm} \mathrm{H}_{2} \mathrm{O}$ were seen with low tidal volumes. In this subgroup of patients, low VT and frequency resulted in alveolar hypoventilation and worsening respiratory acidosis, while adjusting minute volume ventilation upwards was associated with a high risk of pulmonary barotrauma. Therapeutic bronchial lavage was reserved for this group of patients.

IPPV was continued until the ventilator pressures and arterial blood gases were within normal ranges. Weaning was usually readily achieved after a short period of assisted positive pressure breathing or spontaneous breathing with the endotracheal tube in situ.

The indications for therapeutic bronchial lavage were either respiratory acidosis $(\mathrm{pH}<7.2)$ or peak ventilator pressures greater than three times the predicted normal level. The aim of the treatment was to reduce airway obstruction to the point where alveolar ventilation sufficient to maintain normocapnia could be achieved without dangerously high ventilator pressures. Two methods of bronchial lavage were used but no differentiation between the methods is made in analysing data. Additional anaesthesia was given using ether, which causes both bronchodilatation and bronchorrhoea, to assist in the removal of plugs. ${ }^{4}$ Patients were manually ventilated with $100 \%$ oxygen. Aliquots of $10-20 \mathrm{ml}$ of $1.26 \%$ sodium bicarbonate, warmed to body temperature, were injected down the endotracheal tube by an aseptic technique, dispersed by manual IPPV, and removed by postural drainage, physiotherapy, and suction. Since 1980 the sodium bicarbonate solution has been introduced and removed by fibreoptic bronchoscopy, allowing uniform distribution. The procedure was continued until such time as manual IPPV became noticeably easier, usually less than one hour, by which time $350-500 \mathrm{ml}$ had been instilled.

Effective static compliance (C st) was calculated with one equation, $C$ st $=V T /($ Paw $\max -$ PEEP), where VT is the tidal volume, Paw max is the maximum inspiratory airway pressure and PEEP is the positive and expiratory pressure. ${ }^{5}$ None of our $\stackrel{\overrightarrow{\vec{S}}}{\vec{P}}$ patients received PEEP.

An analysis of the intensive care case notes was made to determine details of pre-existing treatment, $\frac{\overline{\bar{s}}}{}$ duration of asthma, and the history of the acute $\mathbb{\otimes}$ attack. In most patients these data were collected on admission to hospital by the attending doctor. Where IPPV was immediate, details of the attack were gath- $\vec{\circ}$ ered after recovery.

In assessment of the complications of IPPV the cri- $\stackrel{\omega}{-}$ teria used for the diagnosis of respiratory infection $\vec{x}$ were abnormal radiological shadowing in the presence of either fever greater than $38^{\circ} \mathrm{C}$ and increasing leucocytosis or the identification of pathogenic bacteria in sputum culture.

\section{Results}

During the 10 years $(1974-83) 213$ patients satisfied $\frac{c}{\bar{D}}$ the criteria for admission to the intensive care unit for the treatment of acute severe asthma from a total of 1942 admitted to the hospital because of asthma. Thirty two patients required IPPV on 34 occasions (15\% of intensive care admissions and $1.6 \%$ of total hospital admissions). The yearly totals for admission $\overline{0}$ to hospital and the proportion requiring IPPV are shown in the figure. The mean age of patients $\stackrel{\mathbb{Q}}{2}$ requiring IPPV was 39.9 (range 16-72) years. Twenty $\overrightarrow{0}$ three $(72 \%)$ were female. Fourteen patients had 3 intrinsic asthma and 18 atopic asthma. Most patients requiring IPPV had a long history of asthma. Twenty $(59 \%)$ had suffered from asthma for more than five years and $27(79 \%)$ had had the disease for at least one year. In only four $(12 \%)$ patients was the duration of asthma less than one year; in three $\frac{5}{3}$ patients the duration was unknown. Despite the chronicity of their asthma, only nine $(27 \%)$ patients had previously attended any hospital for treatment.

The history of the asthma attack was very variable, $\cong$ its duration ranging from 30 minutes to 21 days $\frac{D}{O}$ (mean 8.6, (SD 8.5) days). In only five instances was the evolution of symptoms less than 24 hours, while $N$ on 17 occasions the period of worsening exceeded seven days.

On 12 occasions IPPV was instituted immediately (less than one hour after admission to hospital), the indications being cardiorespiratory arrest in five $\stackrel{\varrho}{\subseteq}$ patients and grade 4 asthma in seven patients. In the remaining 22 cases the mean duration of hospital treatment before IPPV was 10.9 (13.6) hours. The $\frac{0}{0}$ indications for IPPV in this group comprised wors- $\frac{\mathbb{D}}{\mathbb{D}}$ ening clinical trends despite conventional treatment in $\frac{?}{\mathbb{D}}$ 21 patients and impaired consciousness in one $\varrho$ patient. At the time of IPPV the mean heart rate was? 139 (13) beats/min and pulsus paradoxus was almost $\varnothing$ invariable, being present in 19 of the 21 patients. 


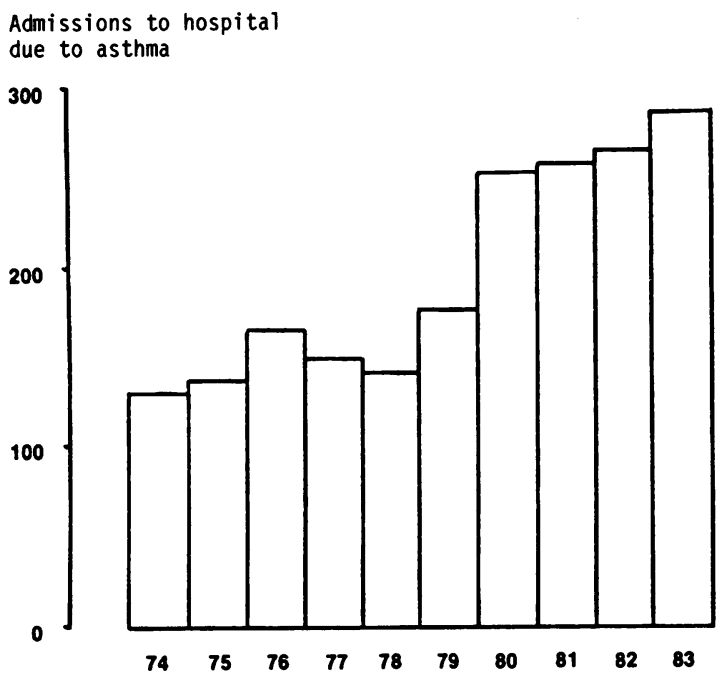

(a)

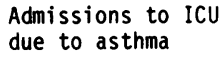

40

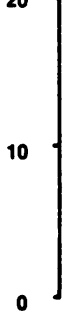

(b)

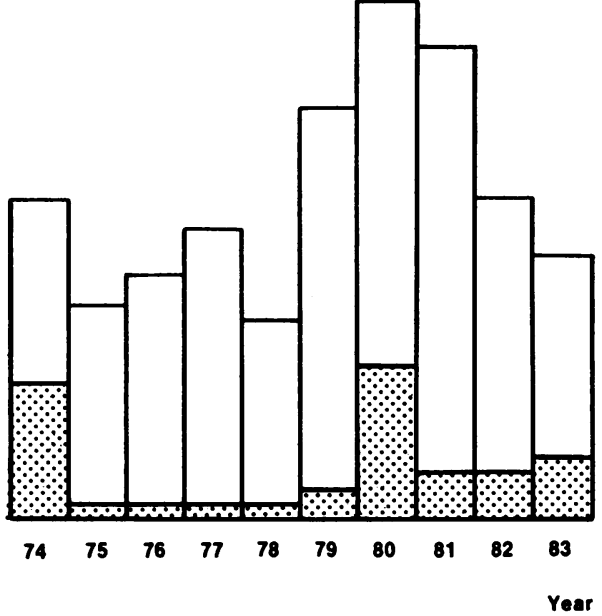

Year

Comparison of (a) the total yearly admissions to Whiston Hospital due to asthma and $(b)$ the proportions requiring admission to the intensive care unit (ICU) and intermittent positive pressure ventilation (hatched).

There was documented hypercapnia (arterial carbon dioxide tension greater than $6.0 \mathrm{kPa}$ ) in 13 patients and normocapnia in five. The mean peak expiratory flow (PEF) was $17 \%$ of the predicted value in eight patients who had values estimated within two hours of starting IPPV.

The mean duration of IPPV was 4.9 (5.3) days (range 17 hours to 23 days). In 21 (63\%) of the 34 episodes of ventilation the duration of IPPV was less than 72 hours. Prolonged IPPV was associated with an increased risk of complications. The complications encountered were: hypotension during induction of anaesthesia in $12(35 \%)$ patients, left pneumothorax in three $(9 \%)$ patients, bilateral pneumothoraces in three $(9 \%)$ patients, pulmonary collapse in three $(9 \%)$ patients, and gastrointestinal bleeding in three (9\%) patients.

Respiratory infection was diagnosed in $12(35 \%)$ patients. The bacteria isolated were Staphylococcus aureus (6 cases), $\beta$ haemolytic streptococcus (4), Klebsiella pneumoniae (2), Pseudomonas aeruginosa (2), Haemophilus influenzae (2), Proteus (1), and Acinetobacter sp (1). Mixed isolates were obtained from five patients.

There were three deaths in this series, all resulting from overwhelming sepsis associated with multiple organ failure. In two of these cases there was a documented respiratory infection, which was the likely source of septicaemia. Both patients had received therapeutic bronchial lavage. The third patient did not receive bronchial lavage and no focus of infection was identified.

In the course of the ten year period under review, a separate study was performed to examine the benefit of high dose corticosteroid treatment in acute severe asthma. The results have been published elswehere. ${ }^{3}$ The number and pattern of complications were similar in both coticosteroid treatment and non-treated patients with the exception of gastric bleeding, which occurred on three occasions, exclusively in patients receiving corticosteroids.

An analysis of pre-existing treatment was made in all ventilated patients. Despite the prolonged evolution of the attack - over 24 hours in $29(86 \%)$ cases and over seven days in $17(50 \%)$ cases-only six patients were receiving oral corticosteroid treatment before admission to hospital. Of these, only two were receiving more than $10 \mathrm{mg}$ prednisolone daily. Seven were receiving inhaled corticosteroid treatment and three inhaled sodium cromoglycate. All had been given $\beta_{2}$ agonists before admission to hospital.

\section{BRONCHIAL LAVAGE}

Therapeutic bronchial lavage was performed 21 times in 19 patients. In all instances there was an improvement in effective static compliance after the procedure from a mean pretreatment figure of 17 (SD 6) $\mathrm{ml} / \mathrm{cm} \mathrm{H}_{2} \mathrm{O}$ to $20(6) \mathrm{ml} / \mathrm{cm} \mathrm{H}_{2} \mathrm{O}$ at one hour and 
24 (9) $\mathrm{ml} / \mathrm{cm} \mathrm{H}_{2} \mathrm{O}$ after four hours. These changes were not significant. The procedure was reserved for those patients with more severe attacks of asthma, suggesting that comparison with the patients not undergoing lavage might not be valid. Thus the mean duration of IPPV for the group having lavage was 4.7 days, compared with only 2.2 days for those not having lavage. Eleven of the 19 patients receiving bronchial lavage developed respiratory infections, compared with only one of 13 controls ( $p<0.01, \chi^{2}$ test). All six pneumothoraces occurred in the patients who had lavage, but a possible causal relationship was observed only once. In one patient the pneumothorax preceded bronchial lavage, while in the remaining four patients pneumothorax was from three to 24 hours after lavage.

\section{Discussion}

Patients requiring IPPV for acute severe asthma constitute a high risk group, many of whom would die without medical intervention. We wished, firstly, to examine the characteristics of the patients and the pattern of their asthma in the hope of uncovering specific features indicating high risk and of obtaining information on avoidable factors. Secondly, we wished to examine factors associated with success or failure and development of complications during IPPV.

Most of our patients had suffered from asthma for several years before their life threatening attack. The fact is in keeping with findings from studies of deaths from asthma, in which the most deaths occurred in patients with chronic asthma. ${ }^{6-8}$ Despite the chronicity of asthma, only $27 \%$ of our patients had received previous hospital treatment. This observation suggests either that the disease was mild before the life threatening attack or that both the patients and their general practitioners underestimated the severity of the disease. Hargreaves has shown that up to $77 \%$ of asthmatic patients have a reduced perception of the severity of airway obstruction, while Seaton has emphasised the underuse by doctors of objective measurements of airflow obstruction. 910

On 29 out of 34 occasions (86\%) the life threatening attack evolved over days rather than hours. This implies that there was time in which treatment could have been adjusted to cope with increasing symptoms. Nevertheless, there was evidence of undertreatment in most of the patients at the time of admission to hospital. In particular, only six out of 32 $(18 \%)$ patients were receiving oral prednisolone and only two of these were receiving more than $10 \mathrm{mg}$ daily. We believe that the underuse of oral corticosteroids probably resulted in part from the reluctance of many general practitioners to institute this treatment before hospital consultation. We are, how- $\vec{F}$ ever, unaware of the number of patients who had con- $-\frac{f}{+}$ sulted their own doctors earlier in the attack, when? corticosteroids might have been started. Our results $\frac{\overline{\bar{N}}}{\bar{N}}$ are consistent with the findings of studies of death $\frac{\text { }}{\widetilde{\sigma}}$ from asthma, where undertreatment is cited as the $\propto$ major preventable factor. One major difference, how-œ ever, between our patients and those dying at home $\vec{\circ}$ from asthma is the time of evolution of the attacks. While the natural history of the attack exceeded $24 \vec{\omega}$ hours in $88 \%$ of our group, the natural history of the fatal attack outside hospital is usually much shorter, $x$ being less than 24 hours in $70 \%$ of cases. ${ }^{6-8}$ Con- $\nexists$ ceivably severe asthma has more than one form of pathophysiological development and data drawn from the study of asthma in hospital may not apply to the community.

Pneumothorax occurred on six occasions, repre- $\vec{c}$ senting an incidence of $18 \%$. In all instances pneumothorax was life threatening, being associated with ${ }^{\oplus}$ systemic hypotension and a rapid rise in ventilator $\overrightarrow{0}$ pressures. The pneumothoraces were either left sided $\stackrel{\infty}{\sigma}$ or bilateral, suggesting a common pathophysiology secondary to mediastinal emphysema and not to rupture of lung parenchyma. ${ }^{11} 12$ Although all pneumothoraces in the present series occurred in patients who had bronchial lavage, temporal relationships $\frac{\circ}{\mathbb{Q}}$ suggest a causal association in only one case. Patients $\vec{\Rightarrow}$ treated by lavage manifested higher maximal airway pressures and they were therefore at increased risk of pulmonary barotrauma.

A complication not commonly associated with asthma is gastrointestinal bleeding, which occurred in $\vec{\partial}$ three out of 34 episodes of IPPV $(9 \%)$. Its frequency in previous studies has ranged from zero to $19 \% .^{13-16}$ Several factors contribute to its occurrence, including peripheral venous engorgement sec- $\delta$ ondary to high intrathoracic pressures, the stress response, the presence of a nasogastric tube, and the $ᄋ$ use of corticosteroid treatment. This complication occurred despite the routine antacid treatment. $\mathrm{H}_{2}$ receptor antagonists may also be used prophylactically but their value is unproved in these circumstances. or

Bronchial lavage remains controversial. While we $\tilde{O}$ found a trend towards improving static compliance $\mathrm{N}_{\mathrm{N}}$ after lavage, the change was not statistically significant. Moreover, the procedure appeared to be associated with an increased risk of respiratory infec- $\frac{\overparen{D}}{\mathscr{D}}$ tion. It should be noted, however, that the lavage pa- $\stackrel{?}{?}$ tients who had represented a group with more severe 0 asthma requiring longer periods of IPPV, which itself $\bar{O}$ may predispose to infection. Recent work suggests $\stackrel{\mathbb{D}}{\circ}$ that the use of ether alone may be equally effective. ${ }^{17} \mathbb{\mathbb { D }}$

The overall mortality rate in the present study was $\frac{\Omega}{\sigma}$ $9 \%$, which compares favourably with that in other series. ${ }^{13-16}$ All three deaths resulted from over- 
whelming sepsis, two of these originating from a primary respiratory infection. Thus IPPV may have been a contributory factor. The best solution remains the avoidance of IPPV by earlier intervention in worsening asthma. Most patients in this series deteriorated over several days before reaching life threatening stages, suggesting that there had been time in which to alter treatment. Yet at the time of admission treatment was inadequate for the severity of the asthma in most patients. Failure to recognise the severity of asthma by both doctors and patients seems likely to be an important factor, indicating the need for education patients and the adoption of objective measurements by doctors.

\section{References}

1 Crompton GK, Grant IWB, Bloomfield P. Edinburgh emergency asthma admission service: report on 10 years' experience. $\mathrm{Br}$ Med J 1979;ii:1199-201.

2 Sherwood Jones E. The intensive therapy of asthma. Proc $R$ Soc Med 1971;64:1151-2.

3 Luksza AR. Acute severe asthma treated without steroids. Br J Dis Chest 1982;76:15-9.

4 Coleman AJ. Inhalational anaesthetic agents. In: Churchill Davidson HC, ed. A practice of anaesthesia. London: Lloyd Luke, 1978:211-303.

5 Don HF, Robson JG. The mechanics of the respiratory system during anaesthesia. Anaesthesiology 1965;26:168.

6 Macdonald JB, Seaton A, Williams DA. Asthma deaths in Cardiff, 1963-74: 90 deaths outside hospital. Br Med J 1976;i:1493-6.

7 Ormerod LP, Stableforth DE. Asthma mortality in Birmingham 1975-77: 53 deaths. Br Med J 1980;i:1493-5.

8 BTA Report. Death from asthma in two regions of England. $\mathrm{Br}$ Med J 1982;285:1251-5.

9 Burdow JGW, Juniper EF, Killian KJ, Hargreave FE, Campbell EJM. The perception of breathlessness in asthma. Am Rev Respir Dis 1982;126:825-8.

10 Seaton A. Asthma-contrasts in care. Thorax 1978;33:1-2.

11 Hamman L. Mediastinal emphysema. JAMA 1945;128:1.

12 Field CE. Spontaneous pneumothorax, massive collapse and subcutaneous emphysema complicating asthma. Arch Dis Child 1943;18:197.

13 Scoggin CH, Sahn SS, Petty TL. Status asthmaticus: a nine year experience. JAMA 1977;238:1158-62.

14 Ambiavagar M, Sherwood Jones E. Resuscitation of the moribund asthmatic. Anaesthesia 1967;2:375-91.

15 Sheehy AF, DiBenedetto R, Lefrak S, Lyons HA. Treatment of status asthmaticus: a report of 70 episodes. Arch Intern Med 1972;130:37-42.

16 Iisalo EUM, Iisalo EI, Vapaavuori MJ. Prolonged artificial ventilation in severe status asthmaticus. Acta Med Scand 1969;185:51-5.

17 Robertson CE, Steedman D, Sinclair CJ, Brown D, Malcolm-Smith N. Use of ether in life-threatening acute severe asthma. Lancet $1985 ; \mathrm{i}: 187-8$. 